\title{
Dutch Research on Knowledge-Based Instructional Systems: Introduction to the Special Issue
}

\author{
Jeroen J. G. van Merriënboer
}

\author{
University of Twente
}

In the last decade, research on computer-based instructional systems has been heavily influenced by rapid advances in cognitive science and artificial intelligence, and by remarkable increases in the power of computers. Due to these factors, there has been a shift from traditional computer-based instructional systems to knowledge-based systems in which knowledge representation techniques are used to model the domain to be taught, the thinking and learning processes of the student, and the applied strategies and tactics for instruction and presentation. Knowledge-based instructional systems may take the form of intelligent tutoring systems (ITSs), adaptive help systems, microworlds, simulations with student-sensitive instructional support, and so forth. The knowledge bases of those systems must be suitable for interpretation by inference engines in order to select appropriate pieces of knowledge for making diagnoses, predictions, and evaluations that steer the instructional process.

Amongst many others, two prevailing problems seem to emerge in this field of research. First, large bodies of knowledge that are required for the development of knowledge-based instructional systems are not available in a useful format. For instance, both theories of learning and theories of instruction often display a vagueness and implicitness that obstructs their implementation in knowledge-based systems. What is needed is experimental research that is aimed at the explication of this knowledge. Second, the extreme interrelatedness of the knowledge that must be present in instructional systems places heavy demands on current knowledge representation, storage, and inference techniques. Obviously, more effective conceptual models relating to the architecture and design methodology of knowledgebased instructional systems are needed in order to properly handle their complexity.

This special issue provides an overview of Dutch research on knowledge-based instructional systems. As will be shown in the next section, this research pertains both to experimental research, aimed at the formulation and explication of knowl- 
edge that is required for the realization of those systems, and to conceptual models, design considerations, and guidelines that are aimed at the improvement of their architecture and design methodology. Furthermore, descriptions of concrete knowledge-based instructional systems and experiences with their development are reported. In my opinion, all three approaches are necessary to make further progress in this fascinating field of research.

\section{INTRODUCING THE RESEARCH}

Burns and Parlett (1991) distinguish three dimensions in the design of knowledgebased instructional systems: (a) a domain knowledge dimension, (b) a communication dimension, and (c) an instructional dimension. The projects reported in this special issue mainly relate to the instructional dimension - that is, to the design of student diagnostic modules, instructional and interface modules, and the interactions between them. For ease of reading, the articles have been partitioned in two groups. The central issue of the first four is student diagnosis; the remaining seven articles mainly concern instructional modules, including interface issues.

\section{Student Diagnosis}

In the first article, Monique Jaspers and Ernest van Lieshout of the University of Nijmegen report on an empirical research project involving children solving arithmetic word problems. They provide a catalog of misconceptions, knowledge deficits, and associated errors in this domain, as well as a procedure that may be used to diagnose the knowledge deficits and misconceptions. A conspicuous feature of the proposed diagnostic procedure is its product-oriented approach; it only takes the student's answers as input and does not require further information on the course of the problem solving process.

The next two articles on student diagnosis provide descriptions of concrete instructional systems. Ab de Haan and Tinus Oppenhuizen of the University of Groningen describe SPELLER, which is a system able to diagnose spelling errors in a "second" language (viz., English), explain these errors to the learner, and cooperate with the learner in solving spelling problems. The system is distinguished by its knowledge-poor approach: No specific domain knowledge or student model is specified beforehand to control the interaction with the student. Instead, "interaction contracts" govern a cooperative search process that is aimed at acquiring relevant domain knowledge. Edwin Bos from the Nijmegen Institute for Cognition Research describes "Het Spelraam," which is a system to communicate procedural knowledge about the conjugation and spelling of Dutch verb forms. The distinguished bug classes and the applied error diagnosis techniques are described in detail.

The last article that is concerned with student diagnosis, by Fred Bosman, Jacqueline Hoogenboom, and Geke Walpot from the National Institute of Educational Measurement, takes a somewhat different view on diagnosis. The authors describe a highly interactive computer-based test that is used to measure vocational skills of pharmaceutical chemists' assistants. An important observation is that knowledge-based instructional systems may lead to a fading of borders between training and testing; obviously, this requires new ways of thinking about psychometric measures such as test difficulty, reliability, and validity. 


\section{Instructional Modules}

The first two articles in this group concern the specification of instructional rules for the tutoring of factual information. Frans van Bussel of the University of Tilburg describes a series of experiments on the learning of vocabulary items in a second language (English). The experiments involve the learning of paired-associate word-translation pairs by experienced and novice language learners; they yield several specific instructional design rules for the teaching of vocabulary items. A common aspect of those rules seems to be that they all promote elaboration of the presented factual information. Ala Samarapungavan from the University of Amsterdam and Jos Beishuizen from the University of Leiden describe a study on the learning from expository text that is presented in a nonlinear, hypertext-like format. A comparison with traditional, linear text formats indicates that well-struc'ured hypertext, using a "conceptual map" that represents the relationships between text concepts, can enhance students' inferential reasoning.

The following two papers relate to learning in the problem solving domain. Marcel Veenman, Jan Elshout, and Vittorio Busato from the University of Amsterdam describe an experimental study on the effectiveness of metacognitive instructional support during experimentation in a computer simulation environment for the teaching of principles of electricity. They conclude that domain knowledge and relevant metacognitive strategies should be taught simultaneously, and that instructional support in simulation environments should apply both to buggy behaviors and to relevant actions that fail to appear. Gijsbert Erkens and Jerry Andriessen of the University of Utrecht present a number of analyses of cooperative problem solving dialogues of pairs of students working on different puzzle tasks. They subsequently describe the Dialogue Monitor, which is an instructional module with an architecture that is based on the analyses of dialogue protocols. The Dialogue Monitor should enable computer-based instructional systems to behave as intelligent cooperative systems (ICSs), in which student and system work together as intellectual partners.

The next two articles delineate conceptual frameworks that may be helpful for the design of instructional modules. Yvonne Barnard and Jacobijn Sandberg from the University of Amsterdam present a taxonomy that may be used to analyze tutorial interactions between student and instructional system. Primitive learner and system actions are described at three different levels: a didactic level, a communication level, and a media level. They illustrate their approach by sample analyses on the interactions that occur in three different interactive learning environments, and conclude that their framework may be helpful in the design of instructional modules. The article by Hein Krammer, Rudolf Maaswinkel, and myself is also concerned with interactions between students and instructional systems. However, our focus is on a taxonomy of content elements for introductory programming instruction; these content elements may be used to instantiate the interactions or, in other words, to compose the deliveries that occur between student and system. The basic claim is that the presented approach is useful for defining an instructional planner for introductory programming ITSs that is relatively independent of both the content of instruction and the applied instructional model; examples from two different instructional models illustrate the flexibility of the approach.

In the final article, Al-Noor Ladhani and Italo de Diana of the University of Twente discuss a severe methodological problem one encounters in the field of knowledge-based instructional systems - namely, how to improve the instructional quality of those systems. Traditional approaches such as control-group designs 
or formative evaluations are often not suitable for reaching this goal. Instead, the authors propose to make computer-based instructional systems highly adaptable and offer second authors (i.e., authors involved in the adaptation of the system) decision support in product optimization. The part of the system involved with decision support for product optimization is called the "predictive agent"; its main function is to predict likely learning outcomes from intended adaptations. Procedures for inductive knowledge acquisition are described by which the predictive agent may automatically construct and refine a knowledge base which specifies the relations between system attributes and learning outcomes.

\section{SUMMARIZING AGENDAS}

Obviously, it has not been possible to present an exhaustive collection of all Dutch research projects within this field of research. But I think that the collection of articles in this special issue provides some good exemplars of projects that are currently conducted in The Netherlands. An interesting question pertains to the general conclusions that can be based on the presented projects (assuming that the great diversity allows for such general results). A first conclusion may be that several Dutch research projects are interested in the domain of (second) language teaching. This is not surprising, since Dutch is a language with a small range; the learning of second languages is particularly important for the population of a small country.

A second, more important conclusion pertains to the state of the art of the field. Whereas a number of the presented knowledge-based instructional systems demonstrate inventive domain and diagnostic modules, no concrete systems with fullfledged instructional modules are presented. Instead, research on instructional modules is mainly restricted to experimental research aimed at the accumulation of knowledge that is necessary to build these modules, and to conceptual frameworks relating to their design and architecture. This seems to be in accordance with Merrill, Li, and Jones (1990), who argue that the state of knowledge about instructional design is too immature to represent this knowledge in expert systems. Based on this observation, it may be expected that the focus of research on knowledgebased instructional systems will be more and more on knowledge of instruction.

Two other themes for future research seem to emerge from the presented articles. Those by De Haan and Oppenhuizen and Erkens and Andriessen show a growing interest in intelligent cooperative systems. In contrast to ITSs and open learning environments (e.g., simulations, microworlds), such systems truly cooperate with the student in order to complete the learning task. And as a final theme, there seems to be a growing interest in knowledge-poor approaches. For instance, De Haan and Oppenhuizen show how relevant domain knowledge can be acquired by the system in a cooperative search process with the student, and Ladhani and De Diana describe a predictive agent which is able to accumulate the knowledge that is necessary to improve the instructional quality of the system and to increase students' learning outcomes. Maybe the future will show that the most effective knowledge-based instructional systems have little knowledge to begin with, but construct their own knowledge bases during their interaction with students. As some people may argue: "In the same way as human teachers do."

Acknowledgments - I would like to thank Martijn Berger, Hein Krammer, and Hans van der Meij for their assistance in reviewing some of the articles that appear in this special issue. 


\section{REFERENCES}

Burns, H., \& Parlett, J. W. (1991). The evolution of intelligent tutoring systems: Dimensions of design. In H. Burns, J. W. Parlett, \& C. L. Redfield, Intelligent tutoring systems: Evolutions in design. Hillsdale, NJ: Erlbaum.

Merrill, M. D., Li, Z., \& Jones, M. K. (1990). Second generation instructional design (ID2). Educational Technology, 30(2), 26-31. 